NOTICE: this is the author's version of a work that was accepted for publication in International Journal of Heat and Mass Transfer.

Changes resulting from the publishing process, such as peer review, editing, corrections, structural formatting, and other quality control mechanisms may not be reflected in this document. Changes may have been made to this work since it was submitted for publication. A definitive version was subsequently published in International Journal of Heat and Mass Transfer, Volume 55, Issues 21-22, October 2012, Pages 6222-6230, http://dx.doi.org/10.1016/j.jheatmasstransfer.2012.06.046 


\section{Capillary Rise in Porous, Fibrous Media During Liquid Immersion.}

Benjamin J. Mullins ${ }^{1,2,3 *}$, and Roger D. Braddock ${ }^{1}$.

1. Atmospheric Environment Research Centre, Griffith University, Nathan QLD Australia 4111

2. Fluid Dynamics Research Group, Curtin University, GPO Box U1987, Perth WA Australia, 6845.

3. Curtin Health Innovation Research Centre, Curtin University, GPO Box U1987, Perth WA Australia, 6845.

Revision Submitted: 17 April 2012

* Corresponding Author

Benjamin J. Mullins

Health Safety and Environment,

Curtin University,

GPO Box U1987,

Perth WA Australia, 6845.

Tel: $\quad+61(0) 892667029$

Fax: +61 (0) 892662358

b.mullins@curtin.edu.au 


\begin{abstract}
The modified Washburn equation has been further developed in this work, to examine capillary rise in porous media which are being immersed in liquid at a continuous rate. The capillary rise rate and/or properties of the media can be determined by a series of differential equations which govern the processes. The model was applied to oleophillic and oleophobic porous, fibrous media with generally good results, however it was found that the model needed to be fitted separately to the initial rise phase and to the longer-term (near-constant slope) phase. It was also found that a good agreement between the theory and experiment could be found if the porous media was contained inside a glass tube so that inflow could only occur from below and not from the sides of the filter. In order to model the insertion of media without this tube, a combination of a horizontal and a vertical capillary model was needed. The model could not be easily fitted to very thin, oleophillic media, and reasons for this are discussed; one likely reason being the inflow of liquid from the sides of the media. However, good agreement between the model and experiment could be found for equivalent media with an oleophobic coating and the same oil.
\end{abstract}

Keywords: Porous media, Capillary rise, Washburn equation, Liquid immersion, Filter 


\section{Introduction.}

Capillarity in fibrous media is a subject which has received a significant amount of study, particularly in recent years [1-5]. However, the capillarity of media composed of loosely packed, near random mats of fibres, such as those found in fibrous filters, has received significantly less attention than more ordered structures.

There exists some works on capillarity of fibrous, porous systems - within palm tree fibres [6], within paper [7], and within filter papers [8]. While these works have provided some illumination to the field, no simple relationship between capillarity and typically measured porous media/filter properties has been presented. Other work purported to model fluid flow through simulated "random" fibre webs [9]. However, the structures used were equivalent to that in woven textiles, and thus far from being a 'near random mat of fibres'. The work of Koponen [9], however, did provide an important relationship between a dimensionless permeability parameter, fluid viscosity and media (textile) porosity. However, determination of this porosity parameter relies upon the prior measurement of the permeability coefficient for the media - and a readily applicable method does not yet exist for the determination of this parameter in highly porous random fibrous media.

Previous work by the authors [10], has found that, within such porous, fibrous media, a strong relationship exists between the capillary and a specific surface area term for the media, defined as the ratio of packing density to fibre radius. In this previous work, the modified Washburn equation was applied to capillary rise curves for porous media where the filter media was in stationary contact with the liquid. Fitting parameters were used to determine capillary radius, dynamic contact angle and equilibrium capillary height. Jaganathan et al. [3] 
applied Richards' equation to thin fibrous media, which is likely to provide a better fit to cases where a highly defined wetting front is not present.

However, almost all previous work which could be found by the authors, considered stationary capillaries which were either horizontally or vertically oriented, with or without the application of a pressure head to the liquid (to increase, or decrease, the capillary rise ). Some studies did extend to the examination of the wetting of non-uniform or irregular capillaries $[11,12]$. There are many industrial applications, such as the manufacture of fibre-composite materials, the coating of textile or fibrous material with liquids (e.g. waterproof coatings), industrial 'belt' filters and other separation processes where the textile or fibrous material is non-stationary, and is being continually immersed into (or withdrawn from) a liquid reservoir. While the wetting of single fibres by immersing them in liquid, or drawing them out of liquid, has received significant study [13], the same cannot be said for more complex structures of fibrous or porous media [14].

The aim of this work is to develop a suitable theory for the capillary rise in a capillary, group of capillaries, or porous media that is being continually immersed in liquid. This model will then be applied to results obtained from a range of low packing density, porous, fibrous media which were immersed in a range of mineral oils with the capillary rise rate studied during the immersion process. 


\section{Capillary Models}

\subsection{Modified Washburn Equation}

Reed and Wilson [15] derived a form of the Washburn equation for rise in a capillary tube, taking account of the forces of inertia (or acceleration), surface tension, viscous drag and gravity. In this derivation, the capillary was assumed to remain stationary, and there was no pressure difference between the inside and outside of the capillary.

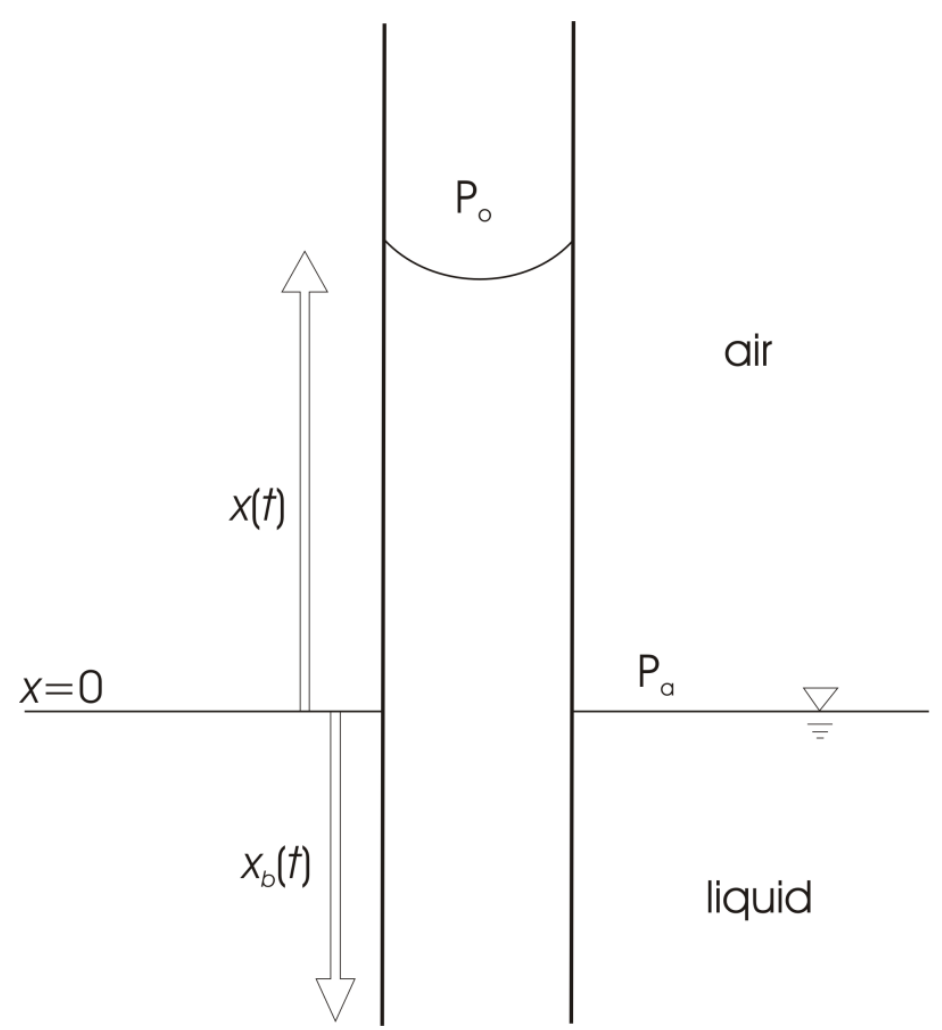

Figure 1 - Schematic of capillary rise within a moving tube. The 'mouth' of the tube is located at the bottom of the two vertical lines, where fluid flows in as the tube descends.

Now consider the situation in Figure 1, where a capillary tube is moving vertically, and the fluid is subject to a pressure difference between the inside and outside of the tube. The height of the meniscus, or the capillary rise, is $x(t)$ taken as positive upwards, while the position of 
the bottom of the tube is $x_{b}(t)<0$, and this varies with time. Both are measured relative to the height of liquid in the external reservoir, which is assumed constant. This assumption is valid when the surface area of the reservoir is large compared to the cross sectional area of the tube. The external pressure on the surface of the fluid reservoir is $P_{a}$, while the pressure on the liquid in the capillary is $P_{o}$, and these are assumed constant. In some cases, $P_{o}$ may be different to atmospheric pressure $P_{a}$, and is retained here for generality. The liquid in the tube is subject to surface tension at the upper meniscus, and this is given by $C T \cos (\theta)$, where $T$ is surface tension per unit length, $\theta$ is the contact angle, and $C=2 \pi r$ is the wetted perimeter of the tube, where $r$ is the radius of the tube [15].

The other forces on the liquid column in the capillary tube are as listed by Reed and Wilson [15], but need to be modified to account for the moving tube. The gravitational force on the length of the fluid column in the tube, is given by $\operatorname{A\rho g}\left(x-x_{b}\right)$, where $\rho$ is the density of the fluid, $g$ is gravity, and $A=\pi r^{2}$ is the cross sectional area of the tube. The excess pressure difference between the bottom of the tube and the meniscus, is given by,

$$
\Delta P=\left(P_{a}-\rho g x_{b}\right)-P_{o},
$$

and this acts vertically. The viscous drag term arises from the relative motion between the tube and the column of liquid. Using an approximation of stream-line flow inside the tube, the Hagen-Poiseuille law gives the total fluid drag $F_{D}$ as,

$$
F_{D}=8 \pi \eta\left(x-x_{b}\right) \frac{d\left(x-x_{b}\right)}{d t}
$$


where the derivative term represents the velocity of the liquid relative to the tube. Applying Newton's laws of motion to the liquid in the tube, leads to the 1-D force balance equation as

$\frac{d\left[A \rho\left(x-x_{b}\right) \frac{d x}{d t}\right]}{d t}=A P_{a}-A P_{o}-A \rho g x+C T \cos (\theta)-8 \pi \eta\left(x-x_{b}\right) \frac{d\left(x-x_{b}\right)}{d t}$,

where the time dependence of $x_{b}(t)$ is yet to be specified. Note that the nonlinear acceleration term is equivalent to a variable mass problem, similar to rocket projectiles where the mass decreases. The Hagen-Poiseuille law applies to laminar flow, which holds when the motion of the tube is slow immersion or removal. This is a second order nonlinear ordinary differential equation, apparently without a closed form of solution. Its solution is readily found using standard numerical techniques.

The time variable may be rescaled using (following Reed and Wilson [15])

$\tau=t \frac{8 \pi \eta}{A \rho}$

so that the derivative terms become

$\frac{d}{d t}=\frac{8 \pi \eta}{A \rho} \frac{d}{d \tau}$

Applying this to (3) gives, 


$$
\frac{d\left[\left(x-x_{b}\right) \frac{d x}{d \tau}\right]}{d \tau}=\frac{A \rho}{(8 \pi \eta)^{2}}\left[A P_{a}-A P_{0}-A \rho g x+C T \cos (\theta)\right]-\left(x-x_{b}\right) \frac{d}{d \tau}\left(x-x_{b}\right) .
$$

Where the capillary is immersed to a fixed or constant depth, the liquid in the capillary comes to a fixed level where the surface tension, gravity and pressure forces are in balance. This steady state corresponds to $x(\tau) \rightarrow x_{\infty}$, as $\tau \rightarrow \infty$, and corresponds to setting the derivative terms, $\frac{d}{d \tau}=0$, in (6), and leads to

$$
x_{\infty}=\left[\frac{P_{a}-P_{0}+\left(\frac{C T}{A}\right) \cos (\theta)}{\rho g}\right] .
$$

Using the transformed variable

$\psi=x-x_{b}$

the dynamic equation (6) then simplifies to the form

$$
\frac{d\left[\psi\left(\frac{d \psi}{d \tau}+\frac{d x_{b}}{d \tau}\right)\right]}{d \tau}=K\left(x_{\infty}-x_{b}-\psi\right)-\frac{\psi d \psi}{d \tau}
$$

where 
$K=g\left(\frac{A \rho}{8 \pi \eta}\right)^{2}$

is a constant. Where the acceleration terms can be ignored, then the left hand side of (9) is set to zero and the corresponding first order differential equation is of the form

$\psi_{1} \frac{d \psi_{1}}{d \tau}=K\left(x_{\infty}-x_{b}-\psi_{1}\right)$

and its solution is denoted by $\psi_{1}$. The condition for (11) to be valid, is that the second derivative terms in (9) are small, compared to the other terms on the right hand side, so that

$\left|\frac{d}{d \tau}\left[\psi_{1}\left(\frac{d \psi_{1}}{d \tau}+\frac{d x_{b}}{d \tau}\right)\right]\right|<<\left|K\left(x_{\infty}-x_{b}-\psi_{1}\right)\right|+\left|\psi_{1} \frac{d \psi_{1}}{d \tau}\right|$.

Using (11), then yields

$\left|\frac{d}{d \tau}\left[K\left(x_{\infty}-x_{b}-\psi_{1}\right)+\psi_{1} \frac{d x_{b}}{d \tau}\right]\right|<<2 K\left|x_{\infty}-x_{b}-\psi_{1}\right|$.

This differential inequality depends heavily on $x_{b}(t)$, through its position, velocity and acceleration. For the special case of the capillary about to start (in close proximity to the surface however inflow not yet commenced), then $x_{b}(t=0)=0, x=0, \psi_{1}=0$ and $x_{\infty}$ does not exist. In this case, the right hand side of (13) is zero. It should be noted that in this case, the modified Washburn equation is not applicable. 
For $x_{b}(t=0)<0$, then the capillary is in contact with the liquid, $x_{\infty}$ does exist but $x(t=0)$ needs to be specified as part of the initial conditions. Then (13) requires knowledge of $x_{b}(t)$ before it can be applied.

Where $\frac{d x_{b}}{d \tau}=v$ is a constant speed, then (13) yields,

$\left|\frac{d}{d \tau}\left[-K v+(v-K) \frac{d \psi_{1}}{d \tau}\right]\right|<<\left|2 K\left(x_{\infty}-x_{b}-\psi_{1}\right)\right|$,

and on simplifying, gives

$$
\left|(v-K) \frac{d^{2} \psi_{1}}{d \tau^{2}}\right|<<K\left|\left(x_{\infty}-x_{b}-\psi_{1}\right)\right| \text {. }
$$

The initial conditions at $t=0$, on $x_{b}(0)$ and $x(0)$, will determine the initial value of $\psi_{1}$. Experimental difficulties may be inherent in obtaining the desired values of these initial conditions. Using $x_{b}(0)=x(0)$, gives $\psi_{1}(0)=0$ and the acceleration terms must be considered. The condition is most readily satisfied when $v \doteq K$, and the second derivative $d^{2} \psi_{1} / d \tau^{2}$ need not be considered unless it is infinite.

As the experiments being conducted in this work utilise a constant velocity of immersion or of removal of the fibrous media from the fluid, it is appropriate to take,

$$
x_{b}=-x_{o}-v \tau,
$$


where $x_{o}$ is the depth of the base of the capillary at time $\tau=0$, and $v>0$ is the speed of immersion. Then,

$\psi_{1}=x+x_{o}+v t$

and $\psi_{1}$ is smallest for small times, i.e. in the initiation of the capillary rise.

When the velocity of immersion $v=0$, then the above analysis needs to be reworked following Reed and Wilson [15], who obtained the condition

$$
J=\frac{g \rho^{2} r^{4}}{64 \eta^{2}}<<1,
$$

for (11) to be valid. The above results also apply to the withdrawal of the filter, or when $v<0$ , provided that the capillary tube does not leave the liquid reservoir. 


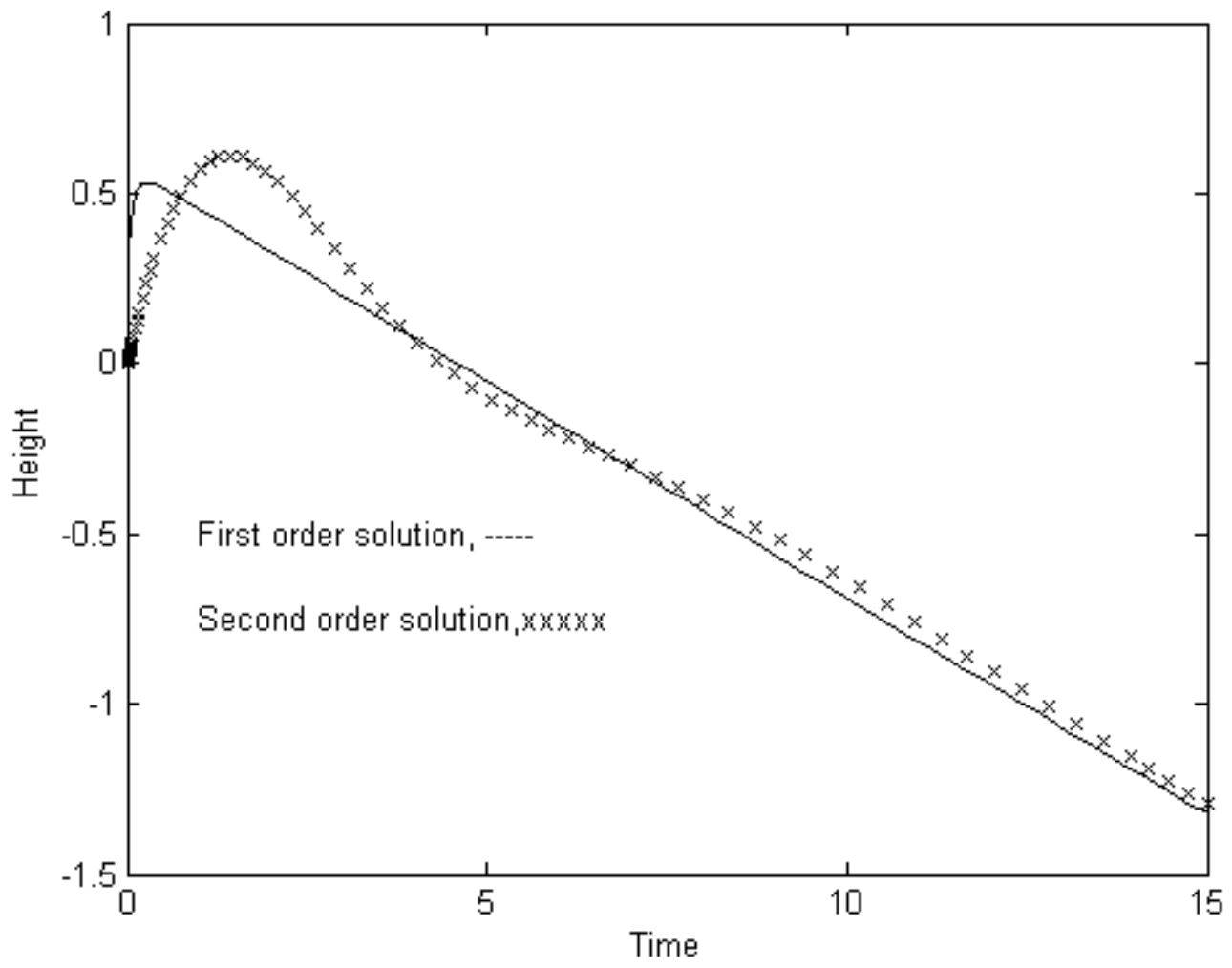

Figure 2 - Numerical solutions of the first order and second order solutions - equations (11) and (9) respectively.

Some numerical solutions showing the solutions of equations (9) and (11) are given in Figure 2, where the initial conditions and the parameter values have been selected to maximise the effects of the acceleration terms. Note that there is no steady state, due to the explicit time dependence through the velocity of the tube. The solution $\psi$ shows some early oscillations, due mainly to the initial conditions required by the numerical integrator. These oscillations quickly die away and the two solutions do converge to a constant velocity for the capillary rise. This is readily seen from the equation for $\psi_{1}$, by assuming that $Q=\frac{d \psi_{1}}{d \tau}$ is constant, and substituting into (11) to obtain, 
$\psi_{1}=\frac{K\left(x_{\infty}-x_{b}\right)}{(Q+K)}$

and on differentiating with respect to $\tau$, then

$Q(Q+K)-K v=0$.

This is a quadratic and has the solutions

$Q=-K / 2 \pm \sqrt{K^{2} / 4+K v}$.

The special case where $K \approx 0$, leads to,

$Q=\frac{d x}{d \tau}+v \approx 0$

and the solution is just a straight line whose position depends only on the initial condition imposed (see below for an example). For the stationary tube, then $v=0$, and there are two roots, $Q=0$, and $Q=-K$ for (20). The case $\frac{d x}{d t}=Q=-K$, can be substituted into (11), and leads to the condition $x=x_{o}$, or $x$ is constant. This solution can only hold for $K=0$. The case $Q=0$, yields $\frac{d x}{d t}=0$, so that the solutions do admit a steady state where it can be shown that $x \rightarrow x_{\infty}$, and that the capillary rise over time $(t)$, can be represented by a logarithmic relationship, of the form [15], 
$t=\frac{8 \eta}{g \rho r^{2}}\left[\left(x_{\infty}+x_{0}\right) \log _{e}\left(\frac{x_{\infty}}{x_{\infty}-x}\right)-x\right]$.

In the absence of a pressure difference then the normal expression for the steady state capillary rise

$x_{\infty}=\frac{C T \cos \theta}{A \rho g}$,

is obtained. This equation is commonly applied to capillary rise problems in industry, including applications involving systems such as textiles or powders, etc. It is reportedly most accurate when a well-defined front exists between the wetted and non-wetted zones [3].

\subsection{Horizontal Capillary model}

The above model can be adapted to consider the movement of liquid in a horizontal capillary tube immersed a distance $z(t)$, in a reservoir. The depth is assumed to vary with time, although it can be considered as a constant as well (see Figure 3). This models a horizontal capillary in a filter being lowered into the liquid reservoir, however a similar approach could be adapted to the drainage of a fully saturated filter media draining when airflow forces are applied [14]. Then the position of the meniscus relative to the origin at one end of the tube, is given by $y(t)$, and the inflow is assumed symmetrical about the middle of the tube at $y=h / 2$, where $h$ is the length of the capillary, or thickness of the filter. At $t=0, y=0$, and, $z=0$, and it is assumed that there is no initial displacement of the fluid in the capillary. Note that relatively thin filters were generally used in the experimental work, compared to the 
vertical displacement of the filter medium. Hence the flow within the filter is primarily horizontal into the filter. Where the filter is slowly lowered into the reservoir, then vertical flows within the filter structure would need to be considered, and the capillary assumption may not be valid.

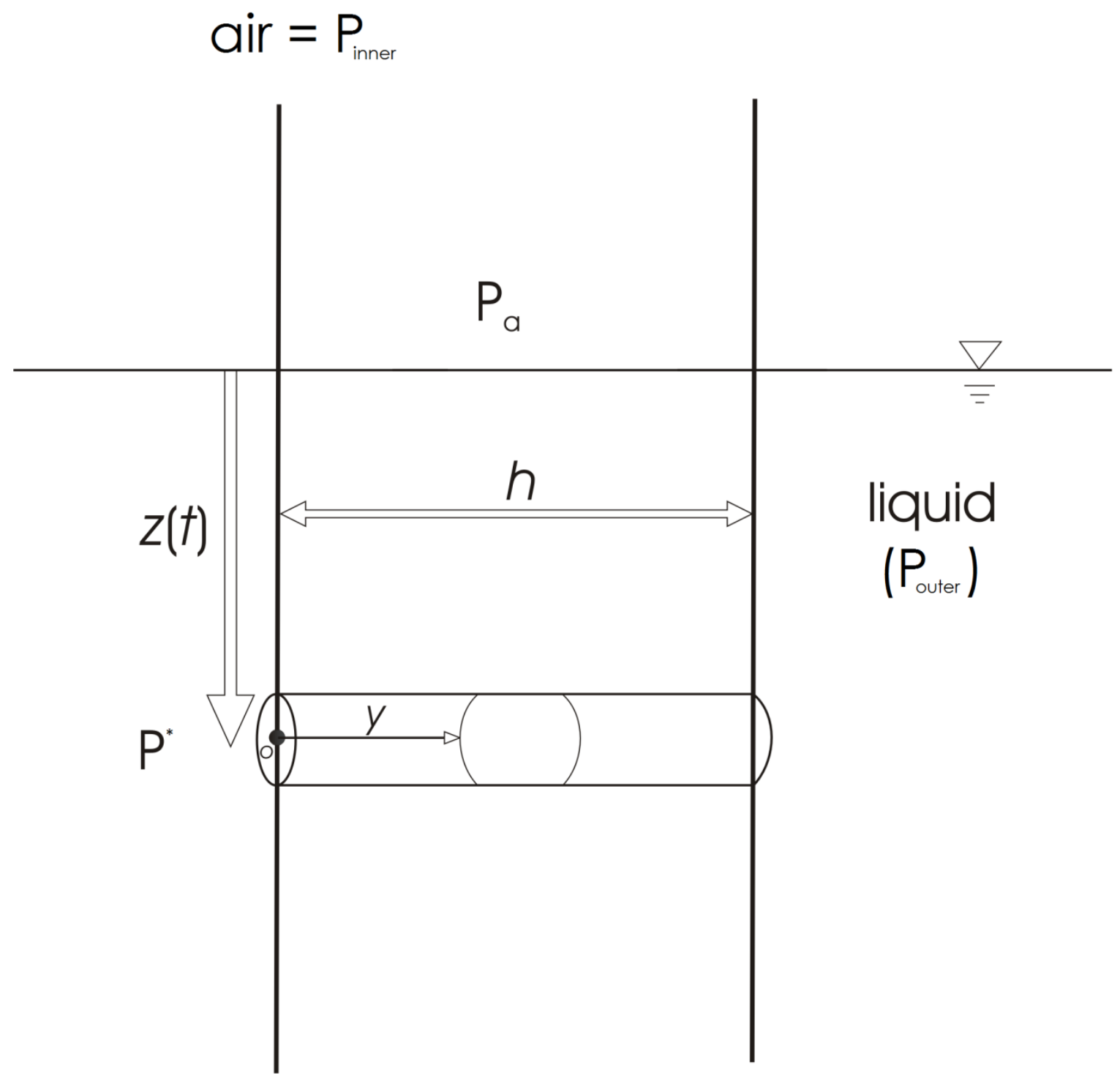

Figure 3 - Schematic of the horizontal capillary model in a filter at depth $z(t)$ below the surface of the reservoir. The filter is located between the two vertical lines.

As the tube, or filter, is lowered into the liquid, the fluid enters both ends and moves under pressure and surface tension forces, toward the centre of the tube. If the capillary surface is 
impermeable to air, then the entrapped air mass cannot escape and is subject to Boyle's Law (pressure* ${ }^{*}$ volume $=$ constant) for the trapped air mass. In the situation being considered, the liquid is permitted to flow along the capillary, but the air is allowed to escape across its boundaries. A full treatment would involve the permeability of the capillary surface, however this is beyond the scope of this study. The displaced air is assumed to be free to flow across the boundary of the capillary, up the filter to the atmosphere. Because of the very low packing density, it is assumed that there is no resistance to this air flow, and that the pressure between the two menisci, is atmospheric pressure $P_{a}$. The pressure on the end of the capillary tube as it is lowered, is $P^{*}=P_{a}+\rho g z$, due to the head of liquid. The force of gravity now enters implicitly through the pressure term, and the explicit gravity term in (3) is not required. Then the force balance equation for the horizontal capillary is

$$
\frac{d\left[A \rho y \frac{d y}{d t}\right]}{d t}=\left(P^{*}-P_{a}\right) A+C T \cos (\theta)-8 \pi \eta y \frac{d y}{d t},
$$

where there is no extension of the tube into the surrounding fluid, i.e., $y_{o}=0$. On ignoring the acceleration terms, this simplifies to

$$
y \frac{d y}{d t}=\frac{A \rho g z(t)-A P_{a}+C T \cos (\theta)}{8 \pi \eta} .
$$

The condition for the acceleration term in (25) to be small is that

$$
A \rho\left|\frac{d}{d t} \cdot\left(y \frac{d y}{d t}\right)\right| \ll|A \rho g z(t)+C T \cos (\theta)|+8 \pi \eta\left|y \frac{d y}{d t}\right|,
$$


Taking $y_{1}(t)$ as the solution of (26), and differentiating (26) with respect to $t$, yields

$\frac{d}{d t}\left[y_{1} \frac{d y_{1}}{d t}\right]=\frac{A \rho g}{8 \pi \eta} \cdot \frac{d z}{d t}$

Substituting (26) and (28) into (27) yields the condition

$$
\left|\frac{d z}{d t}\right| \ll 16 \pi \eta\left|\frac{z}{A \rho}+\frac{C T}{g\left(A \rho^{2}\right)} \cos (\theta)\right|,
$$

for the acceleration terms to be negligible. When $z(t)=z^{*}$, is constant, (26) is easily integrated, and using the initial condition $t=0, y=0$, yields,

$$
t^{*}=h^{2}\left(\frac{\pi \eta}{A \rho g z^{*}+C T \cos (\theta)}\right),
$$

for the time at which the two menisci meet at $y=h / 2$, and the capillary closes. Note the presence of the area $(A)$ of the capillary in (30), indicating that the larger capillaries give smaller values of $t^{*}$, and hence these close first. Smaller capillaries give larger values of $t^{*}$, and hence these close later.

For the moving capillary, it is assumed that $z(t)=v t$, where $v$ is the speed of immersion, and (26) may be integrated to give, 


$$
\frac{y^{2}}{2}=t^{2}\left(\frac{A \rho g v}{16 \pi \eta}\right)+t\left(\frac{C T \cos (\theta)}{8 \pi \eta}\right)
$$

where the initial condition $y(0)=0$, has been used. The time $t_{V}$, at which $y=h / 2$, and the capillary closes, is given by

$$
t_{V}=-\left(\frac{C T \cos (\theta)}{A \rho g v}\right) \pm \sqrt{\frac{(C T \cos (\theta))^{2}}{A \rho g v}+\frac{2 h^{2} \pi \eta}{A \rho g v}}
$$

and the positive sign is chosen to give $t_{V}>0$. Again, the area $(A)$ in the bottom line shows that larger capillaries close first.

The inclusion of the acceleration terms also adds an acceleration time scale to the response. Consider the case $z(t)=z^{*}$, let $\psi=y^{2} / 2$, and on rearranging, (25) reduces to

$$
\frac{d^{2} \psi}{d t^{2}}+\frac{8 \pi \eta}{A \rho} \frac{d \psi}{d t}=\left(\frac{A \rho g z^{*}+C T \cos (\theta)}{A \rho}\right) .
$$

This may be integrated directly to obtain

$$
\frac{d \psi}{d t}+\psi \frac{8 \pi \eta}{A \rho}=t\left(\frac{A \rho g z^{*}+C T \cos (\theta)}{A \rho}\right)
$$

and the integration constant is zero from using the conditions $t=0, y=0$, and $\frac{d y}{d t}=0$. Now (34) may be integrated using the integrating factor method, and 


$$
\frac{y^{2}}{2}=t\left(\frac{A \rho g z^{*}+C T \cos (\theta)}{8 \pi \eta}\right)+2\left(\frac{A \rho g z^{*}+C T \cos (\theta)}{(8 \pi \eta)^{2}}\right) A \rho\left(1-e^{-t \alpha}\right)
$$

after evaluating the integration constant, and where $\alpha=\frac{8 \pi \eta}{A \rho}$. The acceleration time scale is then $t_{A}=\alpha^{-1}=\frac{A \rho}{8 \pi \eta}$, and arises from the exponential term. Where this is small, corresponding to a small $(A)$, the second term on the right hand side in the above equation is small. Then the closure time is given by $t^{*}$ in (30). This corresponds to the small radii capillary tubes. Where $t_{A}$ is large, corresponding to large values of $A$, then the exponential term is asymptotic to zero, and the time to closure depends on the relative size of $t_{A}$ and $t^{*}$. The larger capillaries, with large $A$, take time for the liquid column to accelerate, while small capillaries, with small $A$, accelerate quickly. For small $A$, the exponential term dies out quickly, and the closure is accomplished in time $t^{*}$. For the largest capillaries, the time $t^{*}$ may be attained while the exponential term has yet to decay sufficiently. For intermediate sized tubes, the exponential needs to be included and still represents a reduction in the time to closure. However, the calculation of the closure time is not explicit.

When $z(t)=v t$, a repetition of the above steps leads to the solution

$$
y^{2}=\frac{g v A_{p}}{16 \pi \eta} t^{2}-D t+D\left(\frac{1}{\alpha}-e^{-\alpha t}\right)
$$


where $D=g v\left(\frac{A \rho}{8 \pi \eta}\right)^{2}-\frac{C T \cos (\theta)}{8 \pi \eta}$. The first two terms on the right hand side are the same as in (31) and give a time to closure corresponding to $t_{V}$. The exponential decay term has the same time scale as discussed above. Unfortunately it is not as easy to pick out the separate time scales, but the same pattern applies. For small capillaries with small A, the exponential decays quickly and the time to closure can be estimated from a quadratic in $t$. For large capillaries, the exponential term may not have decayed sufficiently. For intermediate times, the calculation of the time to closure is not explicit. 


\section{Materials and Methods}

\subsection{Experimental}

The experimental apparatus consisted of a microbalance (Model GX-400, AnD, Tokyo), upon which was placed a beaker containing a $100 \mathrm{~mm}$ high column of mineral oil. A sample of fibrous filter media was suspended, above the oil, from a load cell (Model TT100g, Transducer Techniques, California), which was attached to a motorised slide. The accuracy of the balance was $\pm 0.001 \mathrm{~g}$ and the load cell $\pm 0.005 \mathrm{~g}$. The filter sample was lowered at a constant rate of either $0.0071 \mathrm{~ms}^{-1}$ or $0.011 \mathrm{~ms}^{-1}$ until the media was completely immersed. The rate of oil being drawn into the fibrous media from the beaker was then recorded by the load cell (as a mass increase) and the microbalance (as a mass decrease). It was found that both devices returned the same reading for the mass increase/decrease, to the level of resolution of the respective instruments. If the rate of oil drawn into the media was lower than the rate at which the media was being lowered in, then a negative load cell reading and a positive balance reading were recorded. If the rate was faster then the reverse occurred.

Two types of experiments were conducted - experiments where the fibrous material was placed inside a glass tube with a 10mm internal diameter (in order to exclude (or limit) inflow from the sides of the media), and experiments where larger samples of media were used and there was no glass tube. All filter samples which were tested without a surrounding glass tube were rectangular, and ranged in size as well as type of filter material. The fibrous media used in all experiments were composed of loosely packed glass, polymer (polypropylene or polyester), or stainless steel fibres, from a range of manufacturers using a range of different manufacturing methods. The media was usually without any felting, sintering, or binder joining the fibres to each other, except in the case of some of the glass fibre filters which used 
a polymer binder. Mean fibre radius $\left(r_{f}\right)$ ranged between 0.3 and $50 \mu \mathrm{m}$ with the packing density $\varepsilon$, ranging between 0.06 and 0.005 . Many of the filters were composed of polydisperse or bimodally distributed fibre sizes. The width of the samples ranged from 30 $\mathrm{mm}$ to $40 \mathrm{~mm}$, the height from $30 \mathrm{~mm}$ to $90 \mathrm{~mm}$, and the thickness of the media from $0.4 \mathrm{~mm}$ to $15 \mathrm{~mm}$. The filters were separated into two broad groups - the GPSS filters, which were composed of a range of (all) fibre types, however were all thicker, with a lower packing density media, and the TG filters, which were all composed of glass-fibre, much thinner, and with a higher packing density, than the other media. The range of filter properties for each of these two groups is given in Table 1 . Filter material was stored at $20^{\circ} \mathrm{C}$ and $50 \%$ Relative Humidity (RH) prior to the experiments, and experiments were conducted at the same conditions, therefore no possibility existed for the polymer fibres to absorb moisture. All filters were examined using 5 different mineral oil blends with known properties, Sigma Chemicals M3516, M8691, and 3 commercial (screw compressor) lubricating oils, the range of oil properties is given in Table 2 .

All capillarity models use a height of oil in the capillary vs time relationship. Therefore the mass of oil vs time obtained from the experiments was converted to a height based on the measured cross sectional area and packing density of the media. The packing density $(\varepsilon)$ of the media is given by,

$$
\varepsilon=\frac{m_{F} / \rho_{f}}{V_{F}}
$$

where $m_{F}$ is the mass of the filter, $\rho_{f}$ is the density of the fibres of which the media is composed, and $V_{F}$ is the volume of the media. The height of oil within the media was determined by, 
$x(t)=\frac{m_{o}(t) / \rho_{o}}{A_{F c s}(1-\varepsilon)}$,

where $x(t)$ is the height of oil at time $t, m_{\mathrm{o}}(t)$ is the mass of oil at the corresponding time, $\rho_{o}$ is the density of the oil, and $A_{F c s}$ is the cross-sectional area of the media. It is evident that this method assumes that the overall packing density of the filter is constant throughout the media. While this may not be accurate, detailed investigation of the packing density of the media has shown that the average packing density through each horizontal cross-section was relatively uniform.

\subsection{Model Fitting}

The solutions $\psi_{1}$ to the modified Washburn equation need to be fitted to the data obtained from the experiments. This solution has 3 parameters, $K, x_{\infty}$ and $x_{o}$ which need to be fitted to the data obtained from the experiments. The solutions for $\psi_{1}$ are not available in closed form, and the differential equation was solved numerically using the MATLAB ode solver routines. The least squares fitting of the model parameters is done using the error function

$E\left(K, x_{\infty}, x_{o}\right)=\sum_{j}\left(x_{j}^{O}-x^{M}\left(t_{j}\right)\right)^{2}$

where $x_{j}^{O}$ are the observed heights and $x^{M}\left(t_{j}\right)$ are the modelled heights obtained by numerical integration, at times $t_{j}$. The summation is over all of the observed times. 
The MATLAB program fminsearch was used to perform a least squares fit between the model and the data, to obtain the optimal values of the fitting parameters. It was found that the nonlinear nature of the differential equation led to local optima, and the need to have a good first approximate solution before the automated optimiser could be used. This involved considerable experimentation by hand with various initial guesses before suitable parameter values were found. 


\section{Results and Discussion}

\subsection{Experiments without glass tube}

Selected experimental results and corresponding fitted model results are shown in Figure 4 and Table 3. The observations and the fitted curves are shown in Figure 4, for TG06 (the $6^{\text {th }}$ filter in the TG group), for the full data set (Figure 4a), and also for a reduced data set (Figure 4b). The observed data show an initial period of adjustment, followed by a relatively steady change in height with time. This sloping region corresponds to the constant velocity case and led to (15). For (oleo)phillic media, data generally show an initial rise to establish the meniscus, followed by a negative slope, as the capillary rise rate was lower than the rate of insertion of the media (despite it being only $1.1 \mathrm{~mm} / \mathrm{s}$ in the slower case). For phobic systems, the media needed to be inserted sufficiently to initiate imbibition. The fitted parameter values are given in Table 3 for the entire dataset. Figure 4a shows that the longer time behaviour is well captured by the model, while the short time behaviour (Figure 4b) shows significant differences. All of the filters tested showed a similar pattern to these results. There is also a gradual upturn in the observed capillary height as time increases and this is probably due to liquid flowing laterally into the filter.

Figure $4 \mathrm{~b}$ shows a fit to an initial data set representing the short time behaviour. The fit to this part of the data is generally good, and the fitted parameter values are also given in Table 3 for this initial set of data. 


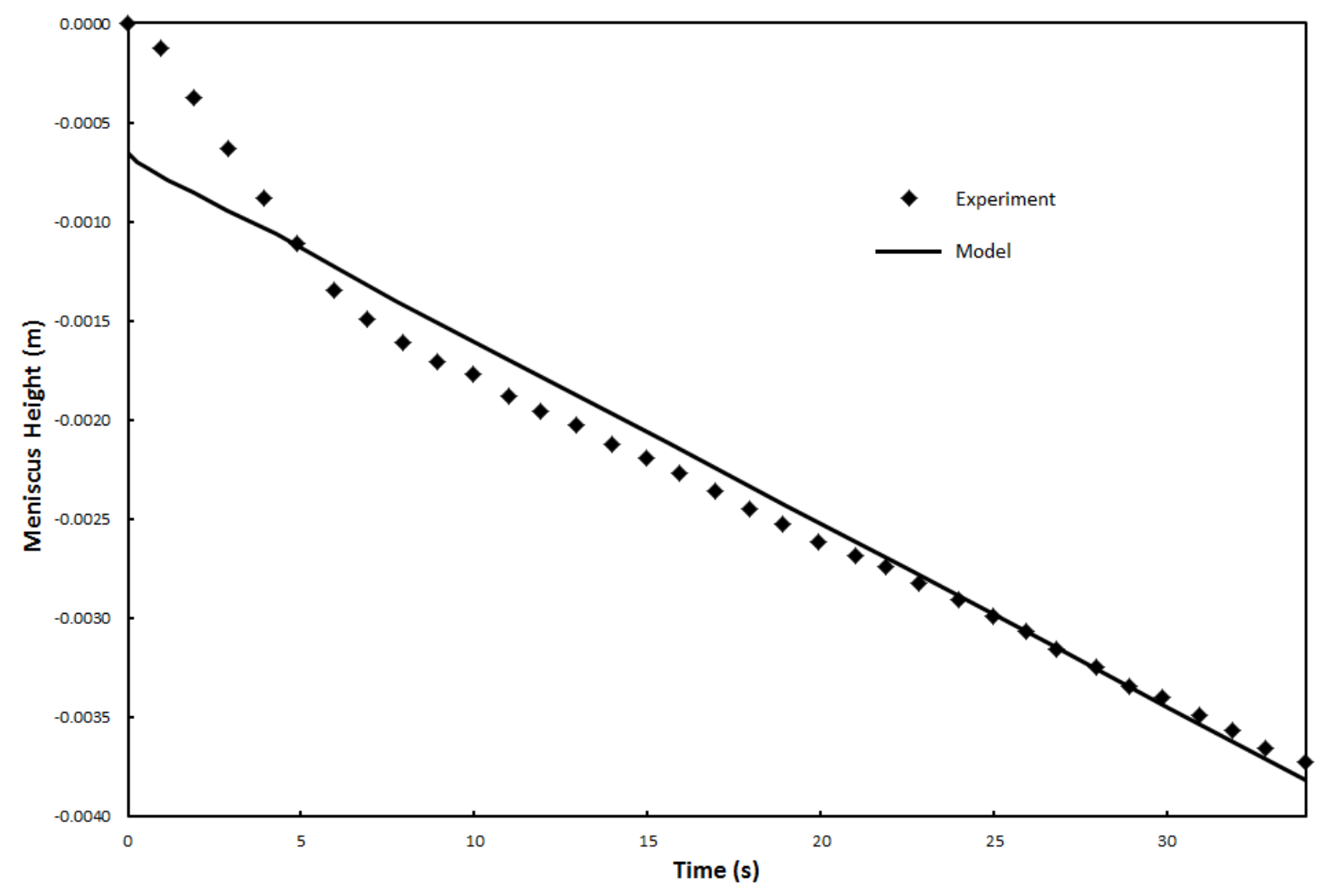

(a)

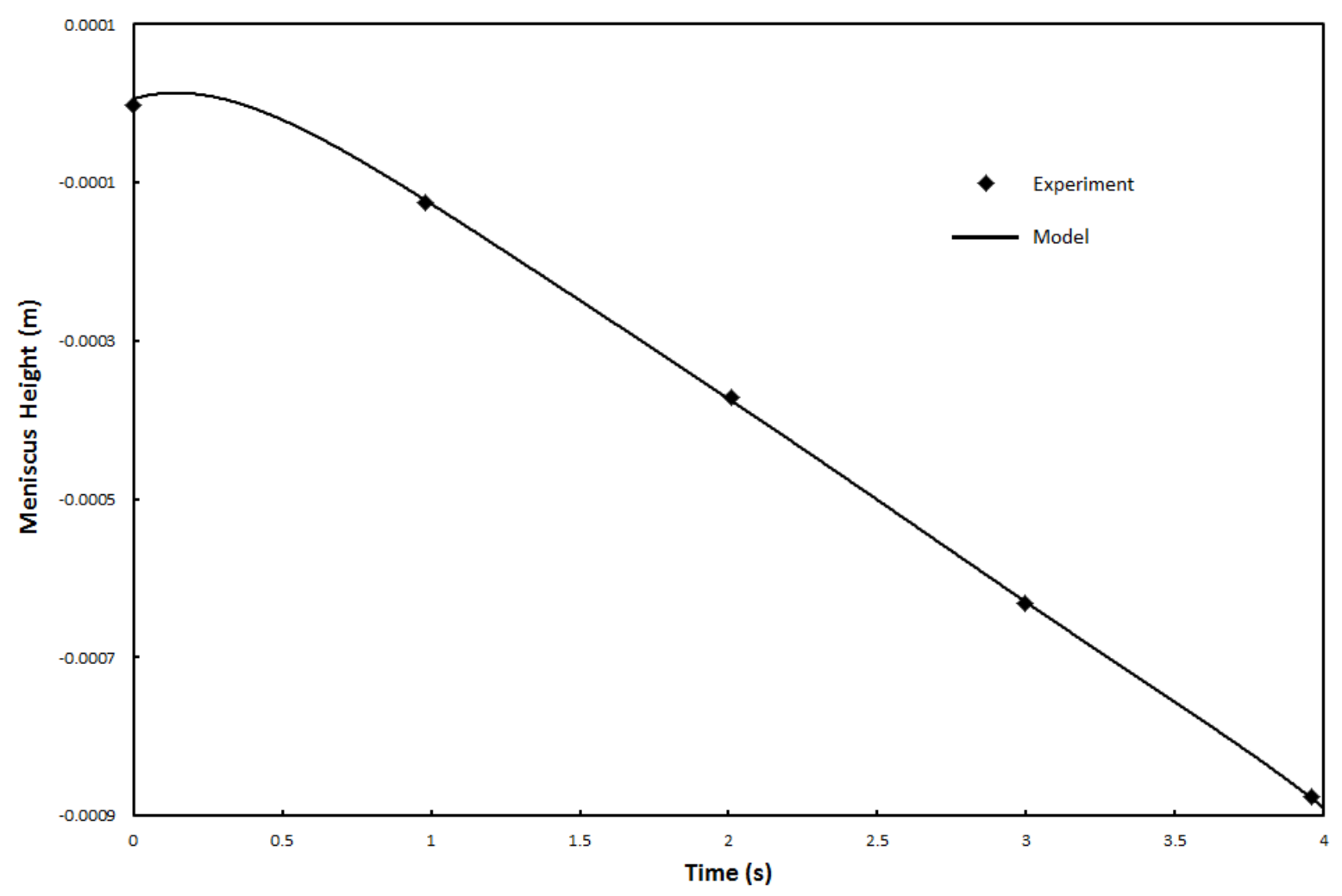

(b) 
Figure 4 - Experimental data for filter TG06, for (a) complete data set and (b) reduced dataset showing initial phase only.

Table 3 shows the parameter values and the least square error, obtained in fitting the data to the modified Washburn model, for the 5 different oils. The sloping section of the curve was used to estimate the value of $K$ using (10), and these values are shown as 'estimated $K$ ' and were used to give an initial estimate of $K$ for the fitting. The fitted values were always less than the estimates, due possibly to the inaccuracies in differentiating the observed data. The values of $K$ also depend on the fourth power of the capillary radius, and are necessarily small. The values of $x_{\infty}$ were negative for the fitting to all of the full data sets, indicating possible contact angles larger than $\pi / 2$. The fitted values of $x_{\infty}$, were positive for the reduced data sets. This sensitivity in the fitted parameter values has been observed by others using the Washburn equation for a stationary capillary rise model [10, 15].The experimental results indicated a relatively poor fit for short time scales. Note that these correspond to the acceleration time scales of the capillaries, and also correspond to the establishment of the flow-field in the fluid and into the capillary. Such effects have not been addressed in the model, and involve much more complex initiation and flow field events. The longer-term effects are obviously well modelled by the Washburn equation.

\subsection{Experiments with media located inside glass tube}




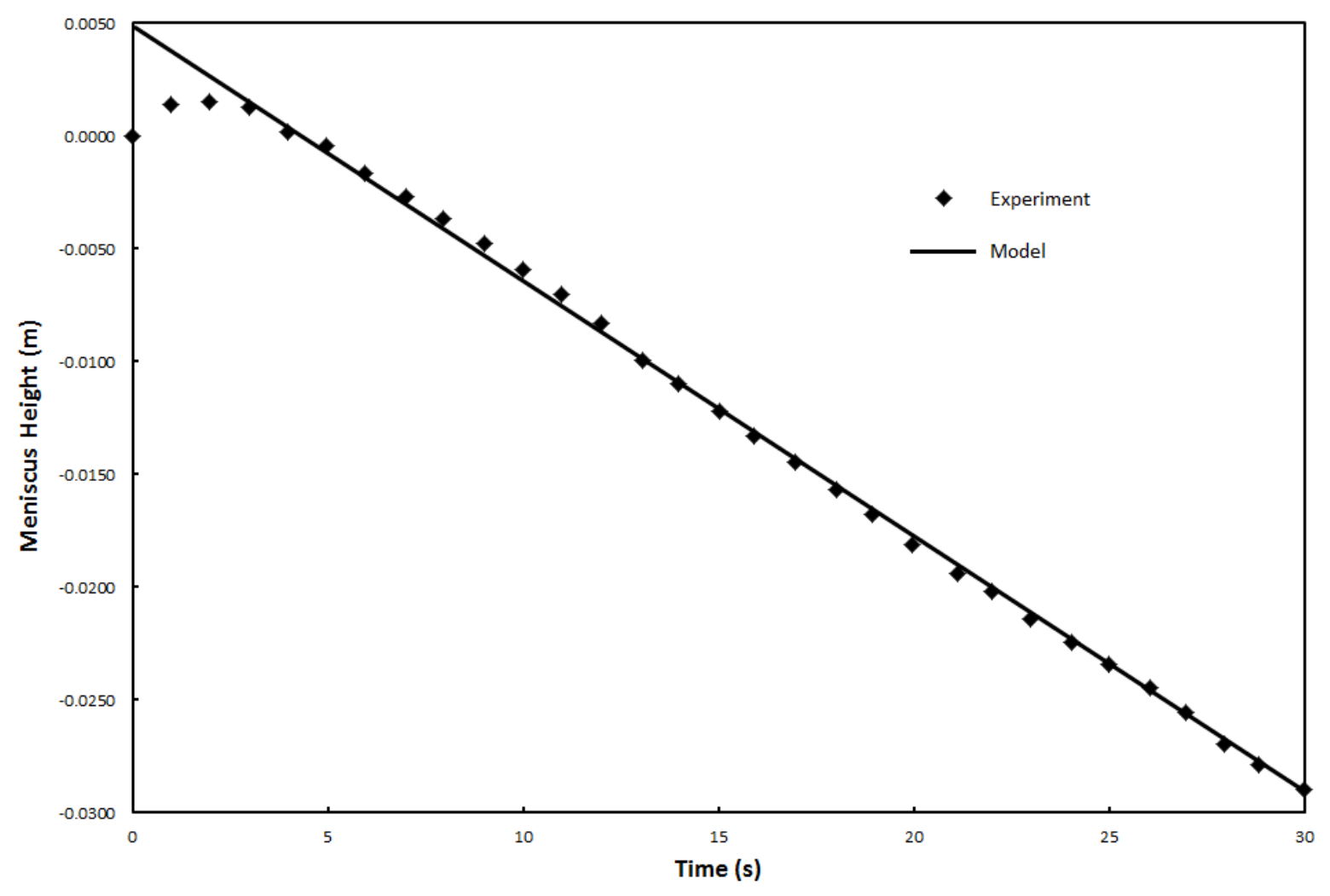

(a)

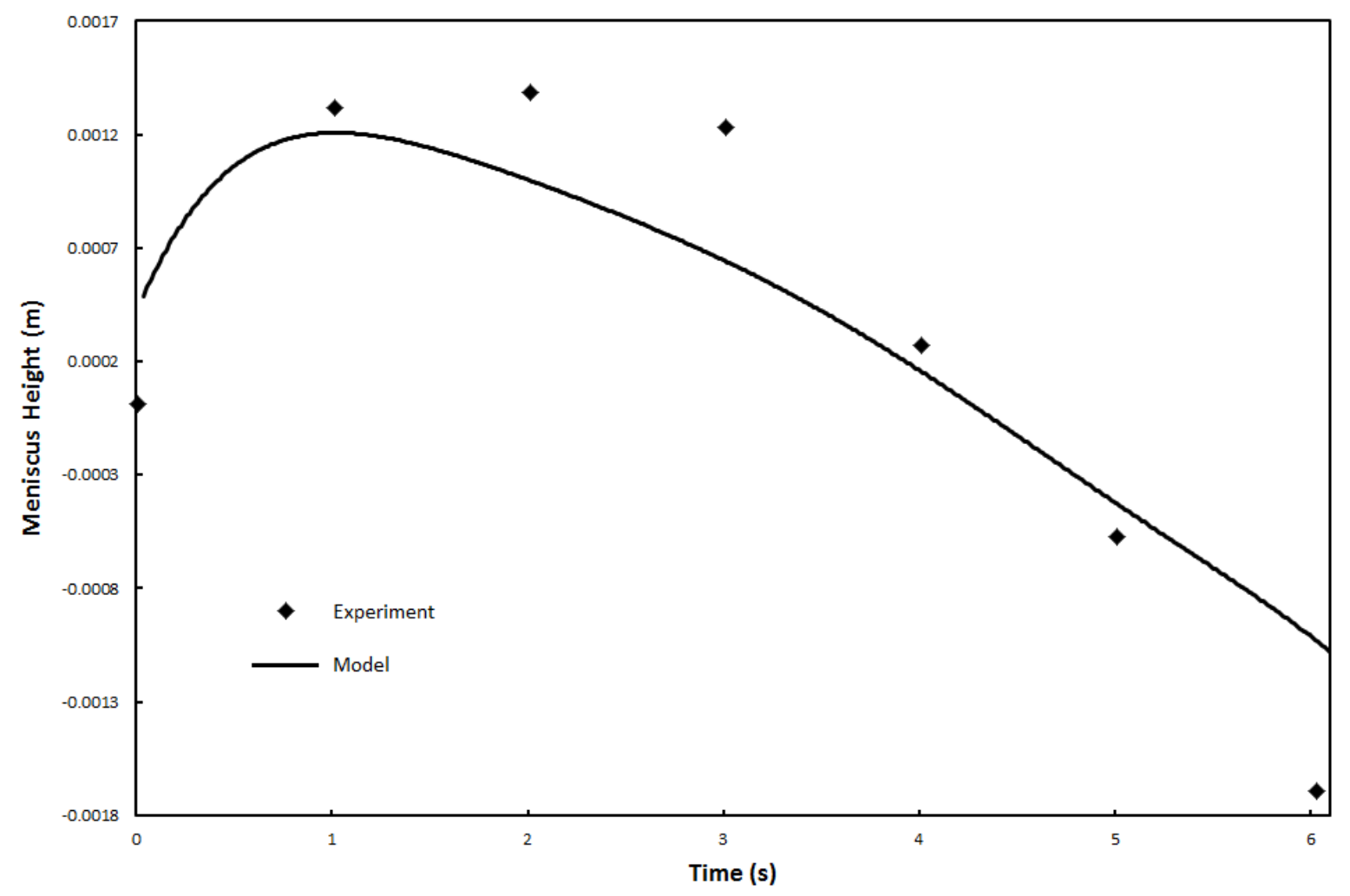

(b) 
Figure 5 - Experimental data for filter GPSS13, for (a) complete data set and (b) reduced dataset showing initial phase only. $V=0.0011 \mathrm{~m} / \mathrm{s}$.

During the fitting of the model to the experimental data, it was found that the model fitted relatively poorly to the initial phase of wetting (e.g. Figure 4a), and the wetting data for some filters with low packing density had significant variations in the rate of wetting. These effects were believed to be due to inflow from the submerged sides of the filter, possibly in combination with localised variations in the packing density of the filter. For this reason, some experiments were conducted using a cylindrical filter sample, placed inside a glass tube with an internal diameter of $10 \mathrm{~mm}$. It was found that when the glass tube was present to stop (or at least minimise) flow in from the sides of the submerged media, a much better fit could be obtained to the data, as shown in Figure 5a for the whole time scale and $5 \mathrm{~b}$ for a shortened time period.

For one of the last set of experiments with a glass tube and a filter, the fitting processes returned very small values of $K$. An extremely good fit was obtained by setting this parameter to zero, and adjusting the initial value for the MATLAB ode integrator. The slope of the resultant straight line agreed to with in 2 significant figures with the experimental velocity of immersion, and the fitted curve is shown in Figure 5a. This is an example of the special case discussed in eqns. (14-17). In this special case, no (or negligible) wetting of the porous media occurs, so that the slope of the curve is equal to the velocity at which the media is being lowered into the liquid.

So we can therefore see that the model produces very good results for porous media without inflow from the sides, and reasonable results when inflow from the sides is possible. When there is no tube or casing surrounding the porous media, it is necessary to account for 
horizontal capillary flow from the submerged portion of the media, as discussed in section 2.2.

In general, the $r_{c}$ and $\theta$ values determined via fitting in this work conformed to the relationships given in our previous work [10]. However, the previous experiments could not consider oleophobic media. It was found that $r_{c}$ values determined for oleophobic media were approximately 60 times larger for the whole (All) dataset and 6 times larger for the initial phase. $\theta$ values were found to be approximately 15 degrees greater for phobic media when compared to similar phillic media. Relationships for $K$ could not readily be found from the fitted results. 


\section{Conclusions}

The Washburn model for capillary rise has been modified for application to moving tubes being immersed into, or removed from, a liquid reservoir. The model has been developed for both a vertical and a horizontal tube, and is readily adapted to handle sloping capillaries. The model has been applied to experimental results obtained for various filters and oils in the reservoir. The results show different short term and long term behaviour, particularly in the parameter values obtained by fitting to the data. These relate to different sizes in the capillary spaces in the filter and to initiation of the flow fields. This result stems from the range of effective capillary radii in the filter and agrees with previous applications of the Washburn Model to stationary tubes.

When lateral fluid flow is restricted by a tube, experimental data and model-fit show better agreement. The closure times for the horizontal tube indicate that the horizontal fluid flow cannot be ignored. However, the Modified Washburn, the horizontal tube and the moving tube equation, are essentially one dimensional, and cannot be extended in their current form, to handle multi-dimensional analysis. The flow of liquids in sparse filter materials are yet to be analysed successfully. 


\section{References}

[1] A. Ashari, T.M. Bucher, and H.V. Tafreshi, A semi-analytical model for simulating fluid transport in multi-layered fibrous sheets made up of solid and porous fibers. Computational Materials Science, 50(2): (2010). p. 378-390.

[2] A. Ashari, T.M. Bucher, H.V. Tafreshi, M.A. Tahir, and M.S.A. Rahman, Modeling fluid spread in thin fibrous sheets: Effects of fiber orientation. International Journal of Heat and Mass Transfer, 53(9-10): (2010). p. 1750-1758.

[3] S. Jaganathan, H.V. Tafreshi, and B. Pourdeyhimi, A realistic modeling of fluid infiltration in thin fibrous sheets. Journal of Applied Physics, 105(11): (2009). p. 8.

[4] H. Tan and K.M. Pillai, Fast Liquid Composite Molding Simulation of Unsaturated Flow in Dual-Scale Fiber Mats Using the Imbibition Characteristics of a FabricBased Unit Cell. Polymer Composites, 31(10): (2010). p. 1790-1807.

[5] K. Bal, J. Fan, M.K. Sarkar, and L. Ye, Differential spontaneous capillary flow through heterogeneous porous media. International Journal of Heat and Mass Transfer, 54(13-14): (2011). p. 3096-3099.

[6] M. Tavisto, R. Kuisma, A. Pasila, and M. Hautala, Wetting and wicking of fibre plant straw fractions. Industrial Crops and Products, 18(1): (2003). p. 25-35.

[7] J. Hyvaluoma, P. Raiskinmaki, A. Jasberg, A. Koponen, M. Kataja, and J. Timonen, Simulation of liquid penetration in paper. Physical Review E, 73(3): (2006.

[8] T. Gillespie, The capillary rise of a liquid in a vertical strip of filter paper. Journal of Colloid Science, 14: (1959). p. 123-130.

[9] A. Koponen, D. Kandhai, E. Hellen, M. Alava, A. Hoekstra, M. Kataja, K. Niskanen, P. Sloot, and J. Timonen, Permeability of three-dimensional random fiber webs. Physical Review Letters, 80(4): (1998). p. 716-719.

[10] B.J. Mullins, R.D. Braddock, and G. Kasper, Capillarity in fibrous filter media: Relationship to filter properties. Chemical Engineering Science, 62: (2007). p. 61916198.

[11] T.L. Staples and D.G. Shaffer, Wicking flow in irregular capillaries. Colloids and Surfaces a-Physicochemical and Engineering Aspects, 204(1-3): (2002). p. 239-250.

[12] W.B. Young, Analysis of capillary flows in non-uniform cross-sectional capillaries. Colloids and Surfaces a-Physicochemical and Engineering Aspects, 234(1-3): (2004). p. 123-128.

[13] D. Quere, Fluid coating on a fiber. Annu. Rev. Fluid Mech., 39: (1999). p. 347-384.

[14] A. Bredin and B.J. Mullins, Influence of flow-interruption on filter performance during the filtration of liquid aerosols by fibrous filters. Separation and Purification Technology, 90: (2012). p. 53-63.

[15] C.M. Reed and N. Wilson, The Fundamentals of Absorbency of Fibers, Textile Structures and Polymers .1. The Rate of Rise of a Liquid in Glass-Capillaries. Journal of Physics D-Applied Physics, 26(9): (1993). p. 1378-1381. 


\section{Acknowledgements}

This work has been funded by Australian Research Council (ARC) Linkage Project LP0883877. The authors would also like to acknowledge the MANN+HUMMEL Group for supporting this work, as well as the generous assistance of the staff of the Karlsruher Institut fuer Technologie, Institute for Mechanical Process Engineering and Mechanics - In particular, Prof. Gerhard Kasper.

\section{Tables:}

Table 1 - Ranges of filter/media properties used in experiments

\begin{tabular}{|l|c|l|c|}
\hline \multicolumn{2}{|c|}{ GPSS Filters } & \multicolumn{2}{c|}{ TG Filters } \\
\hline Packing Density $(\alpha,-)$ & $0.005-0.02$ & Packing Density $(\alpha,-)$ & $0.05-0.07$ \\
\hline Fibre Diameter $\left(d_{f}, \mu \mathrm{m}\right)$ & $4.2-45.5$ & Fibre Diameter $\left(d_{f,} \mu \mathrm{m}\right)$ & $0.6-1.3$ \\
\hline Thickness $(\mathrm{mm})$ & $4.2-15.0$ & Thickness $(\mathrm{mm})$ & $0.4-0.7$ \\
\hline $\begin{array}{l}\text { Material: glass, polymer (PP and } \\
\text { polyester), or stainless steel }\end{array}$ & Material: glass - some with oleophobic \\
\hline $\begin{array}{l}\text { Properties: monomodal fibre distribution, } \\
\text { no binder }\end{array}$ & Properties: bimodal fibre distribution, \\
\hline
\end{tabular}

Table 2 - Ranges of mineral oil properties used in experiments

\begin{tabular}{|l|c|}
\hline Viscosity $(\eta$, Pa.s $)$ & $0.03-0.2$ \\
\hline Density $\left(\rho_{o}, \mathrm{kgm}^{-3}\right)$ & $840-965$ \\
\hline Surface Tension $\left(T, \mathrm{Nm}^{-1}\right)$ & $0.028-0.03$ \\
\hline
\end{tabular}


Table 3 - Results for TG06 filter for 5 different oils

\begin{tabular}{|c|c|c|c|c|c|c|}
\hline & & $x_{\infty}$ & $K$ & $S S Q$ & $\boldsymbol{r}_{\boldsymbol{c}}(\mathrm{mm})$ & $\theta\left(^{\circ}\right)$ \\
\hline \multirow[t]{2}{*}{ Oil A } & All & -0.0066 & 0.00124 & 3.52 & 1.17 & 96.5 \\
\hline & Init. & 0.0036 & 0.00024 & 2.33 & 0.51 & 88.4 \\
\hline Oil B - M3516 & All & -0.0047 & 0.00115 & 1.82 & 0.59 & 92.4 \\
\hline (lowest viscosity) & Init. & 0.3399 & $3.0 \times 10^{-6}$ & 0.41 & 0.03 & 81.1 \\
\hline Oil C - M8691 & All & -0.0064 & 0.00113 & 4.48 & 1.45 & 97.8 \\
\hline (highest viscosity) & Init. & 0.0103 & $6.8 \times 10^{-5}$ & 39.48 & 0.36 & 86.9 \\
\hline \multirow[t]{2}{*}{ Oil D } & All & -0.0053 & 0.00110 & 4.03 & 1.30 & 95.7 \\
\hline & Init. & 0.0170 & 0.00048 & 3.28 & 0.86 & 77.8 \\
\hline \multirow[t]{4}{*}{ Oil E } & All & -0.0060 & 0.00114 & 3.39 & 1.10 & 96.1 \\
\hline & Init. & 0.2500 & $0.26 \times 10^{-7}$ & 13.16 & 0.02 & 86.1 \\
\hline & & & \multicolumn{2}{|c|}{ Mean - All } & $1.12 \pm 0.33$ & $95.7 \pm 2.0$ \\
\hline & & & \multicolumn{2}{|c|}{ SD - All } & $0.36 \pm 0.35$ & $84.1 \pm 4.5$ \\
\hline
\end{tabular}

Table 4 - Properties of filters from previous (static) experiments [10].

\begin{tabular}{|lr|cc|}
\cline { 4 - 4 } \multicolumn{1}{l|}{} & & $\boldsymbol{r}_{\boldsymbol{c}}(\mathrm{mm})$ & $\boldsymbol{\theta}\left(^{\circ}\right)$ \\
\hline TG07 - & All & $1.95 \times 10^{-2}$ & 68.9 \\
\hline same as TG06 except without oleophobic coating $\mathbf{h}=\mathbf{0 . 6 m m}$ & Init. & $8.49 \times 10^{-2}$ & 65.4 \\
\hline GPSS13 & All & $6.5 \times 10^{-2}$ & 88.0 \\
& Init. & $1.53 \times 10^{-1}$ & 84.3 \\
& & & 85.4 \\
\hline
\end{tabular}




\section{Figure Captions}

Figure 1 - Schematic of capillary rise within a moving tube. The 'mouth' of the tube is located at the bottom of the two vertical lines, where fluid flows in as the tube descends.

Figure 2 - Numerical solutions of the first order and second order solutions - equations (11) and (9) respectively.

Figure 3 - Schematic of the horizontal capillary model in a filter at depth $z(t)$ below the surface of the reservoir. The filter is located between the two vertical lines.

Figure 4 - Experimental data for filter TG06, for (a) complete data set and (b) reduced dataset showing initial phase only.

Figure 5 - Experimental data for filter GPSS13, for (a) complete data set and (b) reduced dataset showing initial phase only. 